\title{
Colors of the Yellowstone thermal pools for teaching optics
}

\section{J. Shaw, P. Nugent, M. Vollmer}

J. A. Shaw, P. W. Nugent, M. Vollmer, "Colors of the Yellowstone thermal pools for teaching optics," Proc. SPIE 9793, Education and Training in Optics and Photonics: ETOP 2015, 97931 S (8 October 2015); doi:

$10.1117 / 12.2223177$

SPIE Event: Education and Training in Optics and Photonics: ETOP 2015, 2015, Bordeaux, France 


\title{
Colors of the Yellowstone thermal pools for teaching optics
}

\author{
J. A. Shaw*a ${ }^{*}$ P. W. Nugent ${ }^{\mathrm{a}}$, M. Vollmer ${ }^{\mathrm{b}}$ \\ ${ }^{a}$ Electrical and Computer Engineering Department, Montana State University, Bozeman, Montana,

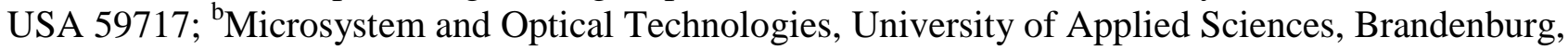 \\ Magdeburgerstr. 50, 14470 Brandenburg, Germany
}

\begin{abstract}
Nature provides many beautiful optical phenomena that can be used to teach optical principles. Here we describe an interdisciplinary education project based on a simple computer model of the colors observed in the famous thermal pools of Yellowstone National Park in the northwestern United States. The primary wavelength-dependent parameters that determine the widely varying pool colors are the reflectance of the rocks or the microbial mats growing on the rocks beneath the water (the microbial mat color depends on water temperature) and optical absorption and scattering in the water. This paper introduces a teaching module based on a one-dimensional computer model that starts with measured reflectance spectra of the microbial mats and modifies the spectra with depth-dependent absorption and scattering in the water. This module is designed to be incorporated into a graduate course on remote sensing systems, in a section covering the propagation of light through air and water, although it could be adapted to a general university optics course. The module presents the basic 1-D radiative transfer equation relevant to this problem, and allows them to build their own simple model. Students can then simulate the colors that would be observed for different variations of the microbial mat reflectance spectrum, skylight spectrum, and water depth.
\end{abstract}

Keywords: Optics education, colors, absorption, scattering, radiative transfer, optics in nature

\section{INTRODUCTION}

Previously, several of us described interdisciplinary education based on optical detectors to notify observers when they could see the Aurora Borealis. ${ }^{1}$ Here we describe a similar interdisciplinary project based on a computer model of the colors observed in the thermal pools of Yellowstone National Park. ${ }^{2}$ More than three million people visit Yellowstone National Park in the northwestern United States each year. When these visitors encounter the world-famous Yellowstone thermal pools and geysers, they naturally wonder what causes the vivid colors. The biologists tell us the colors are a result of different kinds of microbes living in carpet-like mats coating the rock surfaces of the pools. ${ }^{3,4}$ However, these microbial mats really are just the beginning of the explanation and misunderstanding can occur for deeper pools if the optical properties of water are not adequately taken into account. Depending on the temperature of the water, the mats can consist of microbes of a variety of colors, ${ }^{5}$ as summarized in Table 1 and shown in the photographs of Figure 1. At temperatures above approximately $75^{\circ} \mathrm{C}$, the water typically is devoid of microbial mats, leaving a bare rock surface of a grayish-white color. While the information in Table 1 allows visitors to relate the colors they see to the likely pool temperature, this really only works for very shallow water, as scattering and absorption in deeper water significantly alters the spectral distribution of light seen by an observer. ${ }^{2}$ Therefore, an excellent opportunity arises for teaching about the spectrally variable effects of scattering and absorption in water (not entirely different from the same processes in the atmosphere). This teaching can take the form of a simple presentation using photographs and words or a onedimensional computer model to predict how microbial mat reflectance spectra are altered as a function of water depth.

Table 1. Typical microbial mat colors and associated water temperatures in Yellowstone National Park.

\begin{tabular}{|l|l|}
\hline Visible color & Approximate water temperature \\
\hline Brown, red, green & $43^{\circ} \mathrm{C}$ \\
\hline Orange & $57{ }^{\circ} \mathrm{C}$ \\
\hline Yellow, Green, or greenish-yellow & $70^{\circ} \mathrm{C}$ \\
\hline Yellow & $75^{\circ} \mathrm{C}$ \\
\hline Limit to Photosynthesis $75{ }^{\circ} \mathrm{C}$ \\
\hline Pink & $83^{\circ} \mathrm{C}$ \\
\hline White & $85^{\circ} \mathrm{C}$ \\
\hline
\end{tabular}

* jshaw@ece.montana.edu; phone 1406 994-7261; fax 1406 994-5958

Education and Training in Optics and Photonics: ETOP 2015, edited by Eric Cormier, Laurent Sarger Proc. of SPIE Vol. 9793, 97931S · (C) 2015 SPIE, IEEE, OSA, ICO · doi: 10.1117/12.2223177 

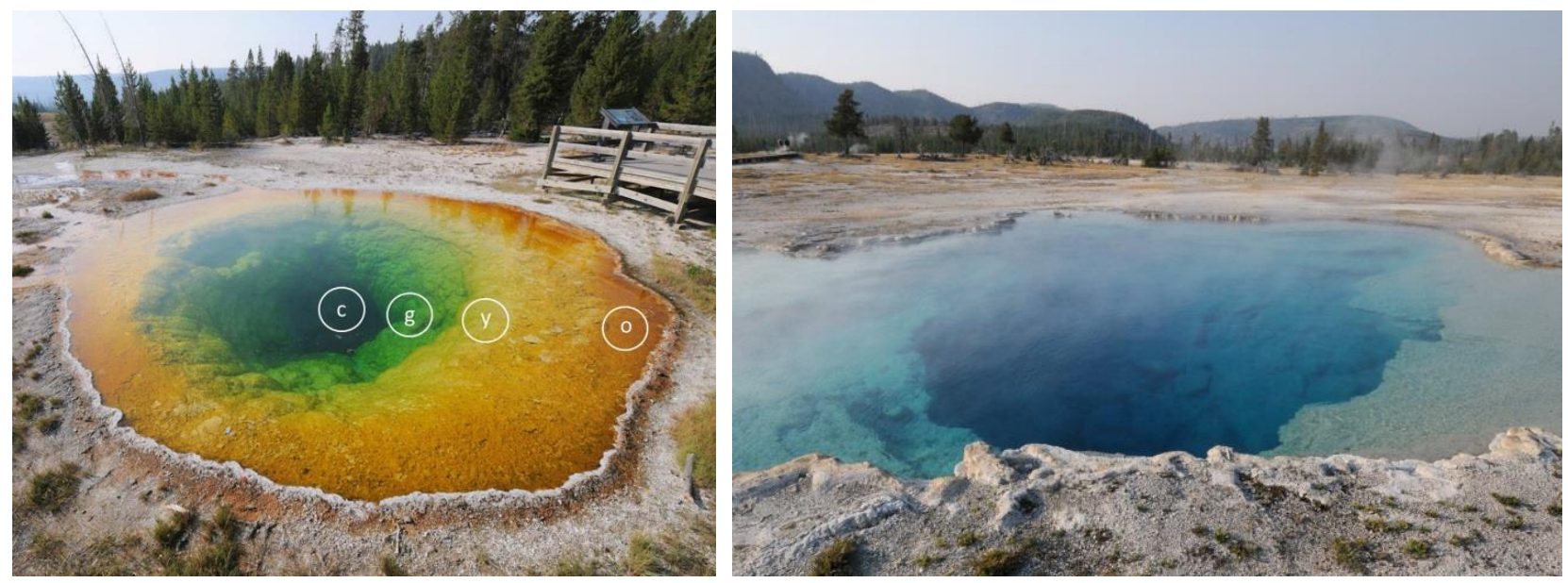

Figure 1. Photographs of (left) Morning Glory Pool and (right) Sapphire Pool at Yellowstone National Park (J. Shaw photos). Labels indicate where spectra were measured for orange (o), yellow (y) and green (g) mats and the center (c).

\section{SIMULATING POOL COLORS STARTING WITH MICROBIAL MAT REFLECTANCE}

If a colored object were slowly lowered deeper and deeper into water, an observer outside of the water would see the color gradually shift toward blue until the object finally disappeared. How much the color would shift until the object disappeared depends on the clarity of the water, but for water with very little suspended particulate matter it would be very significant. This is essentially what explains the color patterns in Morning Glory Pool and similar thermal pools at Yellowstone National Park (see Figure 1). However, instead of an object sinking to the bottom, consider a yellow microbial mat coating the rock walls of a pool like Morning Glory Pool. The color seen by an observer standing next to the pool would fade from yellow near the outer edge to green nearer the center, and finally to dark blue at the very center. Without considering the optical properties of water, Table 1 could lead one to assume that this pattern of colors arose from a temperature gradient within the water (which could, in fact, explain such a pattern in shallower water); however, we recently published computer simulations ${ }^{2}$ closely resembling the left-hand photograph of Figure 1 , which were created using a solid-color surface in a conical-shaped pool such that the mat was seen through progressively deeper water from the outer edge toward the center. The simulation of Morning Glory Pool is shown in Figure 2, the general pattern of which should be compared with the left-hand picture in Figure 1. The orange region at the very outer edge of the photograph in Figure 1 is not included in the simulation because it is almost certainly a different kind of microbial mat because the shallow water at the outer edge is slightly cooler than the deeper water.

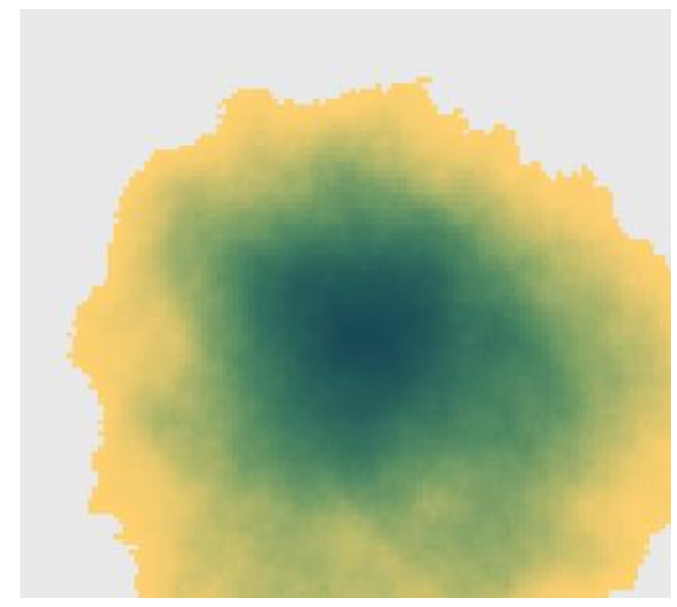

Figure 2. Simulation of Morning Glory Pool using 7-m depth with a single-species yellow cyanobacteria mat whose spectrum was measured in shallow waters near the pool edge. 
Figure 3 shows spectra we measured at each of the locations marked in the left-hand image of Figure 1 (the spectra are all shown normalized to the reflectance of a white Spectralon calibration panel, so the vertical axis is labeled as reflectance). ${ }^{2}$ The yellow reflectance curve, which was measured near the outer edge of Morning Glory Pool at the location marked with the (y) symbol in the left-hand picture of Figure 1, was the starting point for the simulation shown in Figure 2. Therefore, the edges of the simulated pool appear yellow because the shallow water has no appreciable effect. However, as the water becomes increasingly deeper closer to the center of the conically shaped pool, the observed color of the yellow mat viewed through the deeper water becomes green and then bluish-black. This transition in the simulation (Figure 2) matches very closely the actual color pattern apparent in the corresponding photograph (Figure 1).

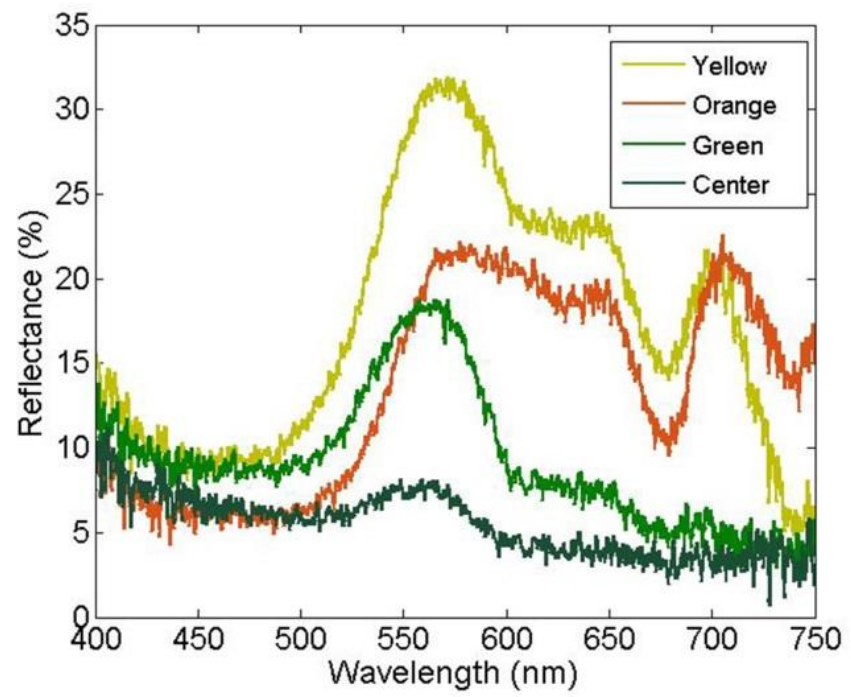

Figure 3. Spectra measured at the positions indicated in Figure 1 ("o" = orange, "y" = yellow, "g" = green, "c" = center).

The depth-dependent color shift that is apparent in both the simulation and the actual Morning Glory Pool photograph is a result of the longer-wavelength light being absorbed and the shorter-wavelength light being scattered out of the beam of sunlight that illuminates the pool (and hence the mat at the bottom of the pool), as indicated by the spectral plots of absorption and scattering coefficients plotted in Figure 4 for pure water. ${ }^{6}$
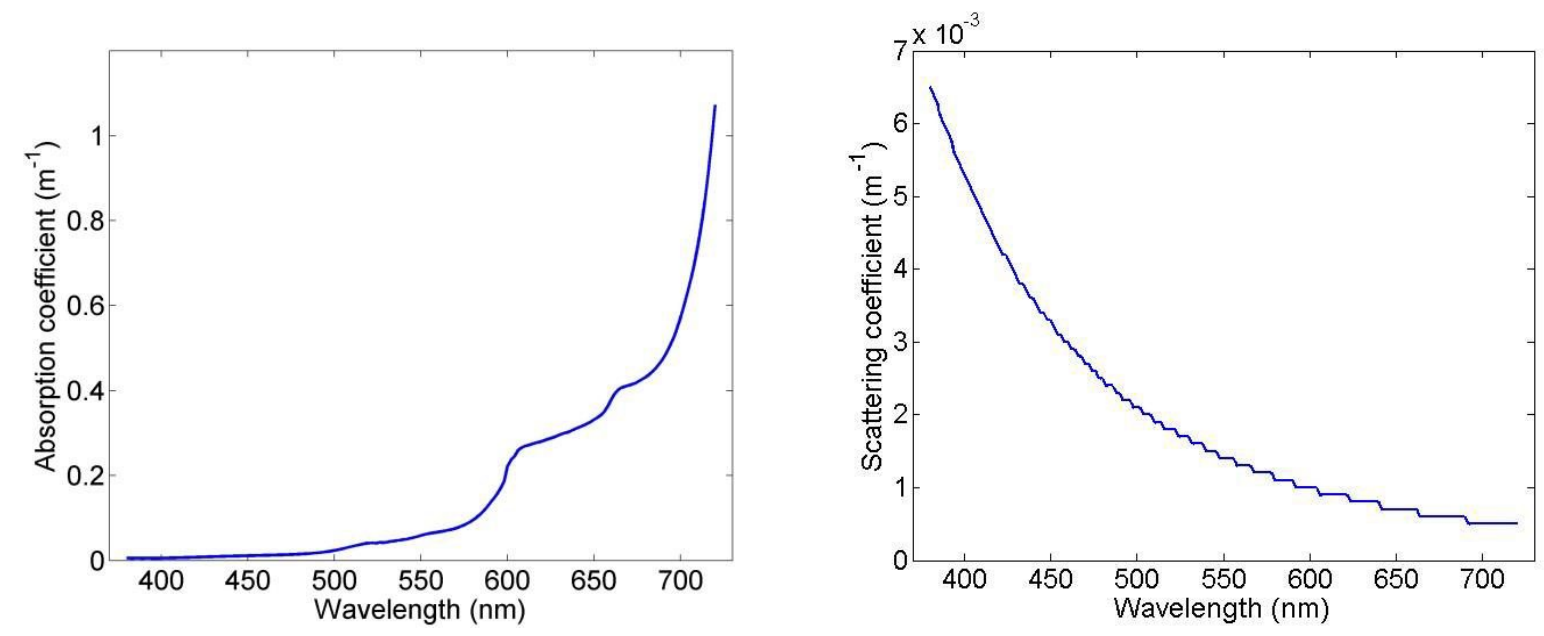

Figure 4. Pure water optical properties: ${ }^{6}$ (a) absorption coefficient $\alpha$ in $\mathrm{m}^{-1}$; and (b) scattering coefficient $b$ in $\mathrm{m}^{-1}$. 
Referring to the geometry shown in Figure 5, the spectral irradiance seen by an observer looking into the pool (at an angle well removed from the solar specular angle) can be written approximately as

$$
E(\lambda)=E_{\text {sky }}(\lambda) R_{w}+E_{\text {sun }}(\lambda)\left(1-R_{w}\right)^{2} R_{\text {mat }} T_{w}{ }^{2}+E_{\text {scattered }}(\lambda)
$$

with

$$
T_{w}{ }^{2}(\lambda)=e^{-\left(a+\frac{b}{2}\right) 2 z}
$$

and

$$
E_{\text {scattered }}(\lambda)=E_{\text {sun }}(\lambda)\left(1-R_{w}\right)^{2} \sum_{n=0}^{z / \Delta l} e^{-2\left(a+\frac{b}{2}\right) n \Delta z}\left(1-e^{-\frac{b}{2} \Delta z}\right) .
$$

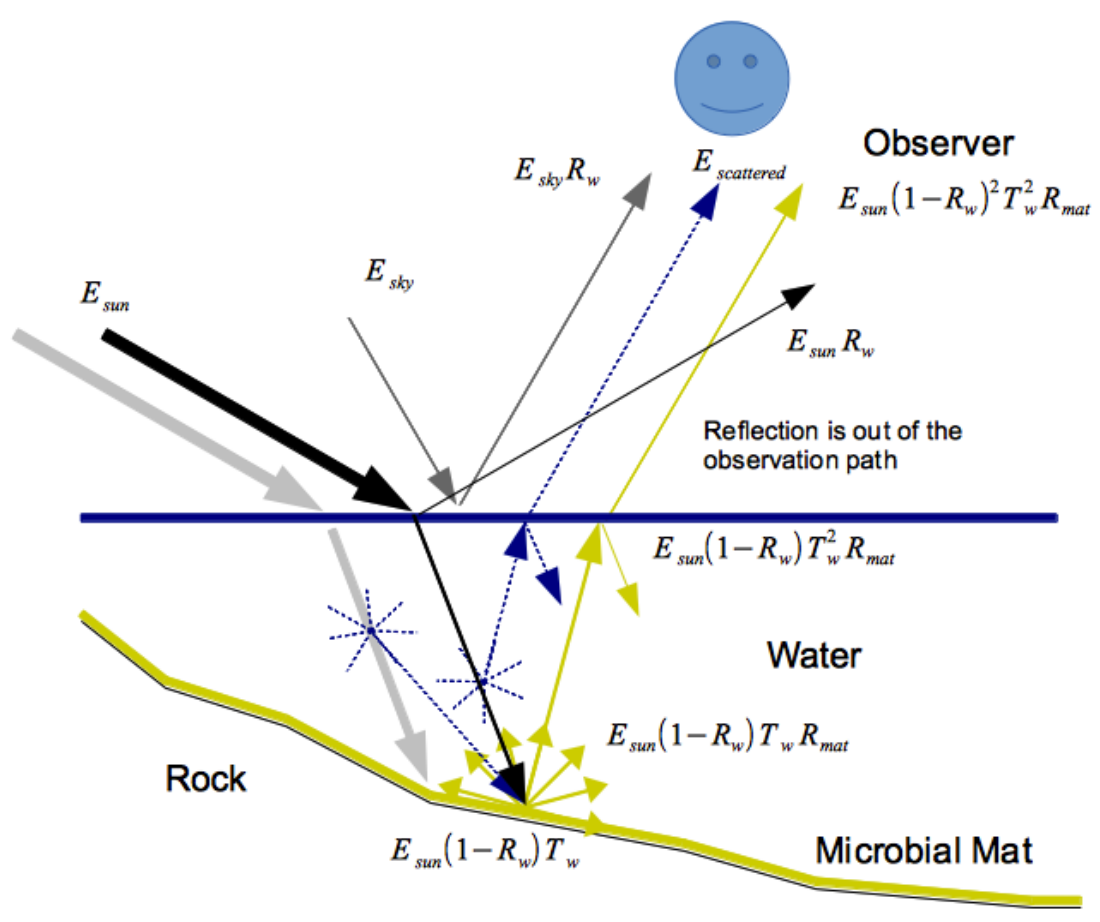

Figure 5. Sketch of the components included in a simple model of observed upwelling spectral irradiance $E(\lambda)$ : diffuse skylight spectral irradiance $E_{\text {sky }}(\lambda)$ reflected from the water (gray arrow); solar spectral irradiance $E_{\text {sun }}(\lambda)$ reflected from the bottom (yellow arrow); and sunlight scattered within the water (dashed blue arrow). For clarity of the description, only a few paths of the upwelling light are shown.

Equation 1 is a simplified 1-dimensional model describing the hemispheric-averaged, upward-directed irradiance leaving the water in terms of the downward solar irradiance and diffuse skylight. The right-hand side of Eq. (1) contains three terms, the first of which is the spectral irradiance of diffuse skylight $E_{\text {sky }}(\lambda)$ reflected from the water with Fresnel reflectance $R_{\mathrm{w}}$ (left-most arrow reaching the observer in Fig. 5).

The second term is direct solar irradiance scattered from the microbial mat into the backward hemisphere with unpolarized spectral reflectance $R_{\text {mat }}(\lambda)$, modified by double-pass attenuation in the water (Eq. 2) with absorption coefficient $a$ along a total path length in the water of $2 z$ (right-hand arrow reaching the observer in Fig. 5). For simplicity, the downward and upward path lengths in the water were both represented by $z$, the scattering in the water was assumed to be equal in the forward and backward hemispheres, and scattering from the mat was assumed to be Lambertian (i.e., scattered radiance equal in all observation angles). Note also that scattering was described by $b / 2$ rather than by the total scattering coefficient $b$ because forward-scattered light contributes to the observed signal and only backscattering leads to attenuation).

Finally, the third term is sunlight scattered in the water (described by Eq. 3 and graphically indicated by the center arrow reaching the observer in Fig. 5). This term is calculated with a multi-layer radiative transfer model in which $\Delta z$ is the 
thickness of the model layer and with $b / 2$ used to approximate the scattering coefficient for light scattered into the backward or forward hemisphere. A useful point to note is that as the downward-propagating light is scattered, the forward-scattered light still contributes to the total illumination at the bottom of the pool. Similarly, as the upwardpropagating light is scattered, the forward-scattered light contributes to the observed light. In Eq. (3) describing the scattered light, the first multiplicative term in the summation, $e^{-2(a+b / 2) n \Delta z}$, describes attenuation along the path between the water surface and the current layer, while the factor 2 accounts for upward propagation back towards the surface

along a path of equal length. The second multiplicative term in the summation, $\left(1-e^{-\left(\frac{b}{2}\right) \Delta z}\right)$, describes scattering within each layer. A sum over all layers down to the depth of the mat gives the total upward-scattered light that would be observed outside of the water. The summation of the three terms (i.e., Eq. 1) gives the total backward-scattered light seen by a pool observer.

We implemented this model using spectra of diffuse skylight and direct solar irradiance from the MODTRAN5 radiative transfer code $^{7}$ with 1976 U.S. Standard Atmosphere profiles ${ }^{8}$ of temperature, pressure, and humidity scaled to match local meteorological measurements at the surface. However, we found the details of the atmospheric calculations not to be particularly important, with the exception that there was a small blue shift in the simulated spectra relative to measurements. We believe this arose because the actual skylight spectrum was spectrally flatter than modeled one, owing to scattering from a modest amount of wildfire smoke in the air, which was not accounted for in the model. ${ }^{2}$ Overall, it is important to note the relative independence of the observed pool colors on the overhead sky spectrum (i.e., the pools would not appear significantly different if viewed on a clear or cloudy day, although the model did not fully consider overcast conditions).

\section{USING THE MODEL TO PREDICT COLORS WITH DIFFERENT PARAMETERS}

One of the most significant benefits of the thermal-pool color model for teaching optical processes is that the model parameters can be changed to see what could happen, even in unusual conditions. One very realistic example of this is shown in Figure 6, which is our previously reported simulation of what Morning Glory Pool might have looked like fifty or more years ago when it was reported to have a higher water temperature (closer to $78{ }^{\circ} \mathrm{C}$, compared with its current value near $69.8^{\circ} \mathrm{C}$ ). ${ }^{2}$ Because the historically reported water temperature was above the threshold of photosynthesis (see Table 1), we simply removed the microbial mat from the model and instead used the spectrum that we measured for the grayish-white rock common to many of the Yellowstone thermal pools. In this particular case, we measured the spectral reflectance of the rocks surrounding Sapphire Pool, currently one of the hottest pools within Yellowstone Park (typically just below the $93{ }^{\circ} \mathrm{C}$ boiling temperature of water at the $2225 \mathrm{~m}$ elevation of this pool above mean sea level). Figure 7 shows a photograph of Sapphire Pool and a plot of the reflectance spectra measured at the dark blue pool center and at the grayish-white rock surrounding the pool.

The blue pool predicted by the simulation (Figure 6) is remarkably different from the multi-colored photograph and simulation of Morning Glory Pool as it appeared in August 2012 (see Figures 1 and 2, respectively). Nevertheless, this blue simulated pool closely resembles the historic descriptions of Morning Glory Pool, which is named for a blue-andwhite flower. The striking similarity between this historic Morning Glory Pool simulation and today's Sapphire Pool is completely expected, since the hot water of the historic Morning Glory Pool would have prevented the growth of a colored bacterial mat, just as we observe currently in Sapphire Pool. We note, however, that despite many sensational reports in the popular press following our original publication, ${ }^{3}$ our study made no attempt to explore the cause of this historic change other than to repeat historic reports that the water became cool enough to support a colored microbial mat as a result of debris partially blocking the vents supplying hot water to Morning Glory Pool. We found that it was a rare reporter who understood and properly explained our curiosity-driven study of these pool colors rather than publishing a sensational description of a fictional study that proved that visitors were ruining the pools. 


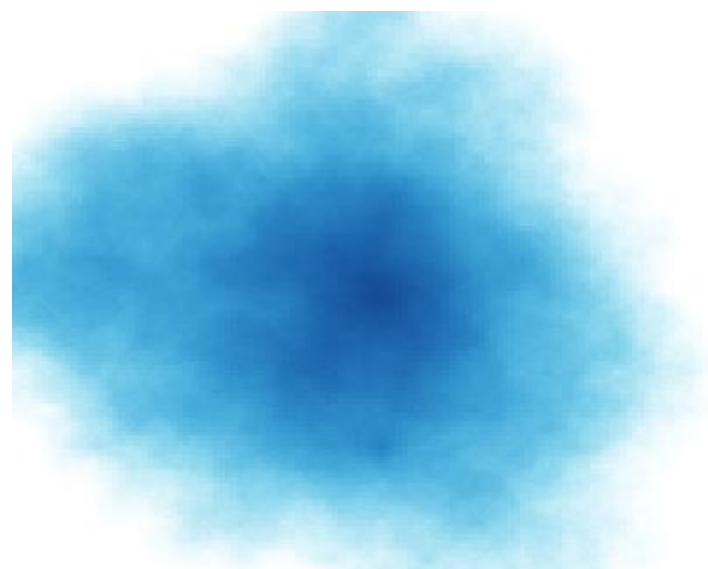

Figure 6. Simulation of Morning Glory Pool with higher water temperature and no microbial mat.

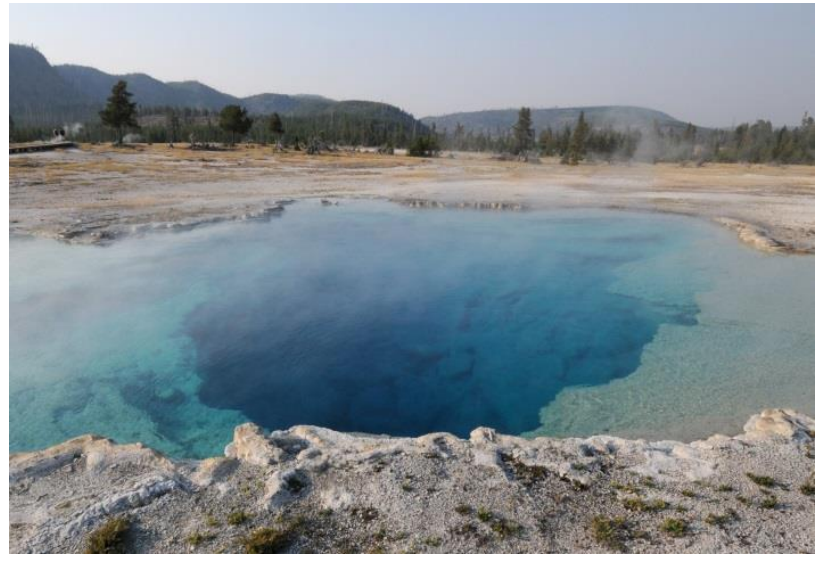

(a)

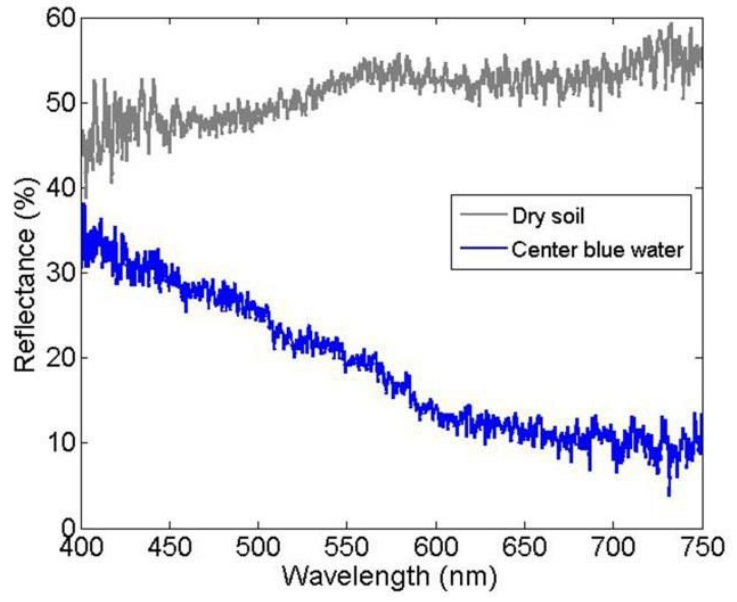

(b)

Figure 7. (a) Photograph and (b) spectra measured for the center of sapphire Pool and the surrounding grayish-white rock.

\section{CONCLUSION}

The popular and beautiful thermal pools of Yellowstone National Park provide ideal examples of colors that are strongly influenced by spectral variations of water absorption and scattering. In shallow water, the observed colors arise simply from the microbial mat coating the underlying rock surfaces, which colors can be related to the water temperature. However, in deeper water, the observed colors are strongly influenced by absorption and scattering in the water. A simple model has been presented, which realistically simulates colors observed in both current and historically reported conditions. To use these simulations to teach about the spectral variation of the optical processes of absorption and scattering in the water and reflection from the microbial mats, we recommend asking students to program the equations given here with an approximation of the water absorption and scattering coefficient curves from Figure 4 . Then the programs can be used with whatever reflectance spectrum the students wish to use as a surface coating (real or hypothetical). Particularly nice results can be obtained with a yellow coating, similar to the microbial mat shown in Figures 1, 2, and 3 for Morning Glory Pool.

\section{REFERENCES}

[1] J. A. Shaw, J. M. Way, N. J. Pust, P. W. Nugent, H. Coate, D. Balster, "Optical aurora detectors: using natural optics to motivate education and outreach,” Proc. ETOP 2009 (https://spie.org/etop/2009/etop2009_2.18.37.pdf). 
[2] P. W. Nugent, J. A. Shaw and M. Vollmer, "Colors of Thermal Pools at Yellowstone National Park," Applied Optics, 54(4), B128-B139 (2015).

[3] D. M . Ward M. J. Ferris, S. C. Nold, and M. M. Bateson, "A natural view of microbial biodiversity within hot spring cyanobacterial mat communities,” Microbiol. Mol. Biol. Rev. 62(4), 1353-1370 (1998).

[4] K. B. De León, R. Gerlach, B. M. Peyton, and M. W. Fields, "Archaeal and bacterial communities in three alkaline hot springs in Heart Lake Geyser Basin, Yellowstone National Park," Frontiers in Microbiology, 4(330), 1-10 (2013).

[5] A. Cox, E. L. Shock, and J. R. Havig, "The transition to microbial photosynthesis in hot spring ecosystems," Chem. Geology 280, 344-351 (2011).

[6] H. Buiteveld, J. H. M. Hakvoort, and M. Donze, "The optical properties of pure water," Proc. SPIE 2258, 174-182 (1994).

[7] A. Berk, G. P. Anderson, P. K. Acharya, L. S. Bernstein, L. Muratov, J. Lee, M. Fox, S. M. Adler-Golden, J. H. Chetwynd, M. L. Hoke, R. B. Lockwood, J. A. Gardner, T. W. Cooley, C. C. Borel, P. E. Lewis, "MODTRAN 5: a reformulated atmospheric band model with auxiliary species and practical multiple scattering options: update," Proc. SPIE 5806, 662-667 (2005).

[8] G. P. Anderson, S. A. Clough, F. X. Kneizys, J. H. Chetwynd, and E. P. Shettle, "AFGL atmospheric constituent profiles (0-120 km)," AFGL-TR-86-0110, Environmental Research Papers, 954 (1986), available from National Technical Information Service, Alexandria, Virginia (www.ntis.gov; also available at http://oai.dtic.mil/oai/oai?verb=getRecord\&metadataPrefix=html\&identifier=ADA175173). 